Division of Geological \& Geophysical Surveys

RAW-DATA FILE 2013-9

\title{
APATITE FISSION TRACK DATA, ALASKA HIGHWAY CORRIDOR, MOUNT HAYES QUADRANGLE, ALASKA
}

\author{
by
}

Diana N. Solie, Paul O’Sullivan, Melanie Werdon, and Richard Lessard

$\$ 2.00$

December 2013

THIS REPORT HAS NOT BEEN REVIEWED FOR TECHNICAL CONTENT OR FOR CONFORMITY TO THE

EDITORIAL STANDARDS OF DGGS

Released by

STATE OF ALASKA

DEPARTMENT OF NATURAL RESOURCES

Division of Geological \& Geophysical Surveys 3354 College Road

Fairbanks, Alaska 99709-3707

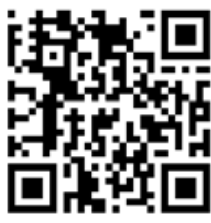






\section{TABLE OF CONTENTS}

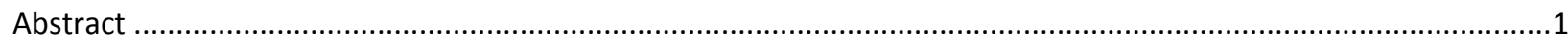

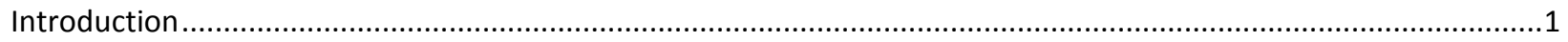

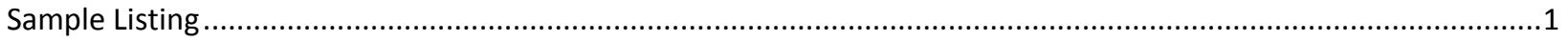

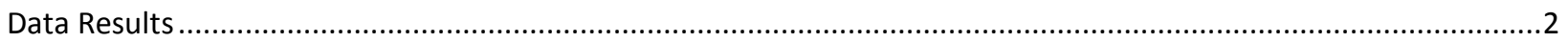

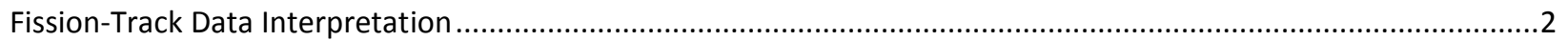

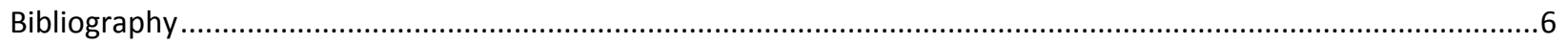

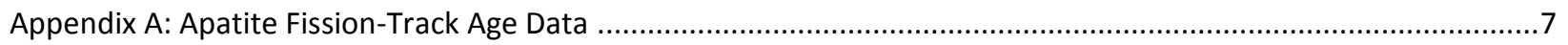

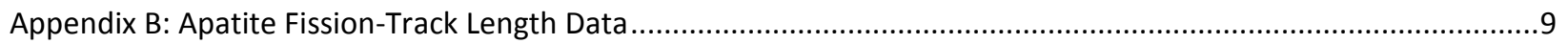

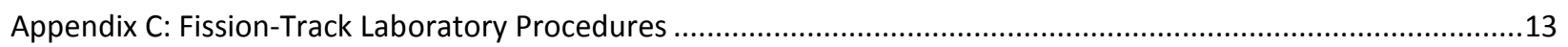

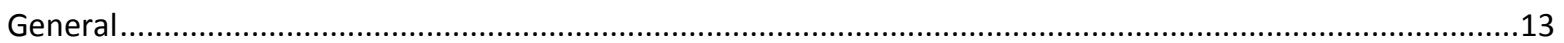

Sample Preparation and Measurement Feasibility Assessment ...............................................................13

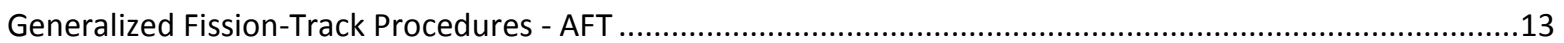

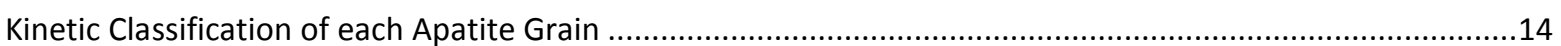

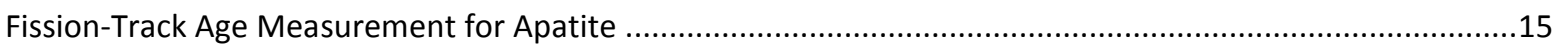

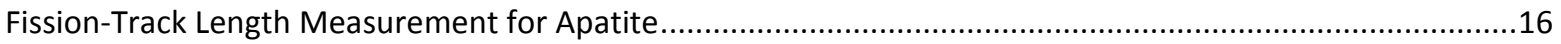

TABLE 1: $\quad$ LISTING OF THE SAMPLES SUBMITTED FOR APATITE FISSION-TRACK ANALYSIS ....................................

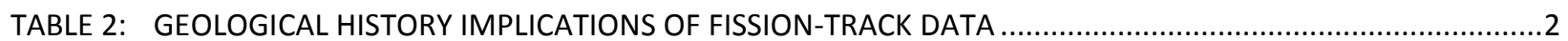

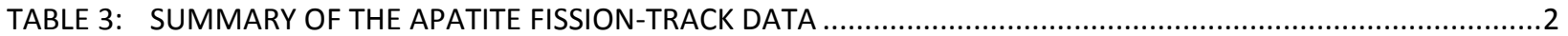

TABLE 4: SUMMARY OF THE APATITE FISSION-TRACK LENGTH DATA (IN MICRONS) FOR ALL APATITE GRAINS COMBINED 



\title{
APATITE FISSION TRACK DATA, ALASKA HIGHWAY CORRIDOR, MOUNT HAYES QUADRANGLE, ALASKA
}

by

\author{
Diana N. Solie ${ }^{1}$, Paul O’Sullivan ${ }^{2}$, Melanie Werdon ${ }^{1}$, and Richard Lessard ${ }^{1}$
}

\begin{abstract}
Two plutonic igneous rock samples were collected from opposite sides of the Sand Lake fault on Macomb Plateau in the Mount Hayes quadrangle, Alaska and analyzed for apatite fission-tracks. The results show a pooled fissiontrack age difference of over $10 \mathrm{Ma}$ between the samples. Modelling suggests that timing of uplift/cooling on the northwest side began $\geq 33.3 \pm 2.4 \mathrm{Ma}$ and on the southeast side began $\geq 59.5 \pm 4.4 \mathrm{Ma}$.
\end{abstract}

\section{INTRODUCTION}

The Alaska Division of Geological \& Geophysical Surveys conducted a multi-year project studying the geology and geologic hazards along the Alaska Highway corridor between 2006 and 2010. As part of the bedrock-geologic mapping, samples were collected for geochronologic analyses using ${ }^{40} \mathrm{Ar} /{ }^{39} \mathrm{Ar}$, apatite fission-track and $\mathrm{U} / \mathrm{Pb}$ dating techniques. Summaries of analytical results appear on bedrock-geologic maps, which are being published in three segments. In the western-most segment, between Delta Junction and Dot Lake, two rock samples were collected for apatite fission-track analysis. This report supplies the data for the apatite fission-track analyses. Sample coordinates are given in NAD27 (North American Datum of 1927) decimal degrees, and will be shown on the bedrock geologic map for this segment of the corridor project (Werdon and others, 2014 in press). The two samples from Segment 1 of the Alaska Highway corridor project were submitted to the Apatite to Zircon, Inc. (A2Z) laboratory, where apatite fission-track analysis was done by Paul O'Sullivan in 2008. The resulting data, excerpted from the laboratory report, are given below and in Appendices A and B. Laboratory procedures are described in Appendix C.

Sample 07MBW1007A was collected from the northwest side of the Sand Lake fault on Macomb Plateau (Werdon and others, 2014 in press). It is a megacrystic alkali feldspar biotite granodiorite with about $7 \%$ biotite. Sample 07RL485A is very coarse-grained biotite granite from the southeast side of the Sand Lake fault on Macomb Plateau. Sand Lake fault is a linear northeast-trending fault that transects the corridor, and is clearly evident as a linear feature in airborne magnetic and electromagnetic resistivity surveys (Burns and others, 2006). No evidence of geologically recent movement has been observed along the trace of the fault (Carver and others, 2008). The purpose of these samples, in conjunction with ${ }^{40} \mathrm{Ar} /{ }^{39} \mathrm{Ar}$ analyses from both sides of the Sand Lake fault, is to provide information about the time and rate of uplift on both sides of the fault.

\section{SAMPLE LISTING}

Table 1 contains a listing of the samples submitted for analysis, the number assigned to each sample in the A2Z laboratory, the amount of apatite isolated from each sample, the number of grain mounts prepared for each sample, and the quality of the apatite grains obtained for analysis.

\footnotetext{
${ }_{1}^{1}$ Alaska Division of Geological \& Geophysical Surveys, 3354 College Road, Fairbanks AK 99709-3707

${ }^{2}$ Apatite to Zircon, Inc., 1075 Matson Road, Viola ID 83872-9709
} 
TABLE 1: LISTING OF THE SAMPLES SUBMITTED FOR APATITE FISSION-TRACK ANALYSIS

\begin{tabular}{|l|l|l|l|l|l|l|}
\hline $\begin{array}{l}\text { Sample } \\
\text { Number }\end{array}$ & $\begin{array}{l}\text { A2Z } \\
\text { Sample } \\
\text { Number }\end{array}$ & Latitude & Longitude & $\begin{array}{l}\text { Apatite } \\
\text { observed }\end{array}$ & $\begin{array}{l}\text { Grain } \\
\text { Mounts } \\
\text { Prepared }\end{array}$ & $\begin{array}{l}\text { Apatite Quality: } \\
\text { 1=poor, } \\
\text { 10=excellent }\end{array}$ \\
\hline 07MBW1007A & $880-20$ & 63.6313 & -144.7647 & $\begin{array}{l}1000 s \\
\text { (excellent yield) }\end{array}$ & 1 & 6 \\
\hline 07RL485A & $880-21$ & 63.6266 & -144.6690 & $\begin{array}{l}1000 s \\
\text { (excellent yield) }\end{array}$ & 1 & 6 \\
\hline
\end{tabular}

\section{DATA RESULTS}

The geologic history implications of the apatite fission-track data are summarized in Table 2. The apatite fissiontrack age data are summarized in Table 3. The apatite fission-track length data are summarized in Table 4.

TABLE 2: GEOLOGICAL HISTORY IMPLICATIONS OF FISSION-TRACK DATA

\begin{tabular}{|l|l|l|l|l|}
\hline Sample Number & $\begin{array}{l}\text { A2Z Sample } \\
\text { Number }\end{array}$ & $\begin{array}{l}\text { Age of Oldest } \\
\text { Apatite Fission } \\
\text { Track (Ma) }\end{array}$ & $\begin{array}{l}\text { Timing of Initiation } \\
\text { of Uplift/Cooling } \\
\text { (Ma) }\end{array}$ & EasyR \\
\hline 07MBW1007A & $880-20$ & $33.3 \pm 2.4 \mathrm{Ma}$ & $\geq 33.3 \pm 2.4 \mathrm{Ma}$ & Not applicable \\
\hline $07 \mathrm{RL} 485 \mathrm{~A}$ & $880-21$ & $59.5 \pm 4.4 \mathrm{Ma}$ & $\geq 59.5 \pm 4.4 \mathrm{Ma}$ & Not applicable \\
\hline
\end{tabular}

TABLE 3: SUMMARY OF THE APATITE FISSION-TRACK DATA

\begin{tabular}{|c|c|c|c|c|c|c|c|c|c|c|c|c|c|c|}
\hline 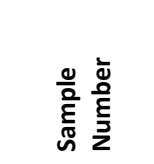 & 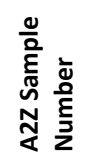 & 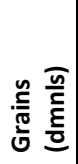 & 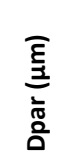 & 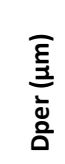 & $\begin{array}{l}\bar{n} \\
\frac{0}{0} \\
\frac{D}{5} \\
z \\
z\end{array}$ & 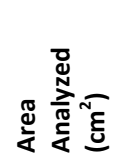 & $\frac{\text { č }}{\text { č }}$ & 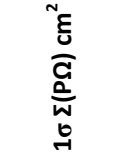 & $\sum_{\mu}^{n}$ & $\sum_{n}^{n}$ & 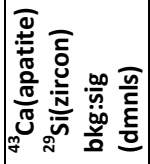 & 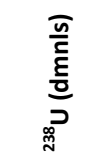 & $\frac{\frac{\pi}{c}}{\frac{\varepsilon}{0}}$ & 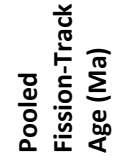 \\
\hline \multicolumn{15}{|c|}{ Age Standards } \\
\hline Durango & DR06 & 492 & 1.52 & 0.29 & 3091 & $2.27 \mathrm{E}-02$ & $7.117 \mathrm{E}-04$ & $1.335 \mathrm{E}-06$ & 14.5149 & 0.2625 & $2.565 \mathrm{E}-02$ & $\begin{array}{l}2.166 \mathrm{E}- \\
03\end{array}$ & 0.9665 & $31.4 \pm 0.8$ \\
\hline \multicolumn{15}{|c|}{ Interior Alaska } \\
\hline 07MBW1007A & $880-20$ & 32 & 1.29 & 0.28 & 251 & $6.63 \mathrm{E}-04$ & $4.14 \mathrm{E}-05$ & $1.29 \mathrm{E}-06$ & 10.8562 & 0.2383 & $2.31 \mathrm{E}-02$ & $1.27 \mathrm{E}-02$ & 0 & $32.9 \pm 2.4$ \\
\hline 07RL485A & $880-21$ & 23 & 1.28 & 0.29 & 269 & $5.11 \mathrm{E}-04$ & $2.89 \mathrm{E}-05$ & $8.28 \mathrm{E}-07$ & 10.6314 & 0.2332 & $3.50 \mathrm{E}-02$ & $6.89 \mathrm{E}-02$ & 0.017 & $49.2 \pm 3.5$ \\
\hline
\end{tabular}


TABLE 4: SUMMARY OF APATITE FISSION-TRACK LENGTH DATA (IN MICRONS) FOR ALL APATITE GRAINS COMBINED

\begin{tabular}{|c|c|c|c|c|}
\hline & \multicolumn{2}{|c|}{ Length Standards } & \multicolumn{2}{|c|}{ Interior Alaska } \\
\hline Sample Number & & & 07MBW1007A & 07RL485A \\
\hline A2Z Sample Number & $\begin{array}{c}\text { Durango-D } \\
\text { (31.4 Ma) }\end{array}$ & $\begin{array}{c}\text { Fish Canyon Tuff } \\
\text { (27.8 Ma) }\end{array}$ & $880-20$ & $880-21$ \\
\hline Tracks $(\mathrm{N}=)$ & 138 & 130 & 125 & 101 \\
\hline Mean, Error $(\mu \mathrm{m})$ & $14.59 \pm 0.08$ & $15.01 \pm 0.09$ & $14.16 \pm 0.16$ & $13.60 \pm 0.18$ \\
\hline Standard Deviation $(\mu \mathrm{m})$ & 0.98 & 1.02 & 1.83 & 1.84 \\
\hline Dpar $(\mu \mathrm{m})$ & 1.87 & 2.36 & 1.3 & 1.26 \\
\hline Dper $(\mu \mathrm{m})$ & 0.22 & 0.46 & 0.2 & 0.17 \\
\hline $0-4(\mu \mathrm{m})$ & - & - & - & - \\
\hline 4-5 ( $\mu \mathrm{m})$ & - & - & 1 & - \\
\hline 5-6 ( $\mu \mathrm{m})$ & - & - & - & - \\
\hline 6-7 ( $\mu \mathrm{m})$ & - & - & - & - \\
\hline $7-8(\mu \mathrm{m})$ & - & - & - & 1 \\
\hline 8-9 ( $\mu \mathrm{m})$ & - & - & 1 & - \\
\hline 9-10 $(\mu \mathrm{m})$ & - & - & 3 & 4 \\
\hline $10-11(\mu \mathrm{m})$ & - & - & 1 & 5 \\
\hline $11-12(\mu \mathrm{m})$ & 1 & 1 & 9 & 10 \\
\hline $12-13(\mu \mathrm{m})$ & 5 & 3 & 6 & 11 \\
\hline $13-14(\mu \mathrm{m})$ & 28 & 16 & 25 & 22 \\
\hline 14-15 $(\mu \mathrm{m})$ & 60 & 46 & 31 & 20 \\
\hline $15-16(\mu \mathrm{m})$ & 32 & 39 & 40 & 24 \\
\hline $16-17(\mu \mathrm{m})$ & 10 & 23 & 8 & 4 \\
\hline $17-18(\mu \mathrm{m})$ & 2 & 2 & - & - \\
\hline
\end{tabular}

\section{FISSION-TRACK DATA INTERPRETATION}

The following pages show how the model results appear when they are loaded into the quantitative modeling program HeFTy (C2004-2012 Apatite to Zircon, Inc. and Richard A. Ketcham). Using this program, it is possible to test alternative thermal histories for each sample while maintaining the analytical results as a background for guidance. HeFTy implements various laboratory calibrations of the behavior of fission tracks in apatite in response to heating and cooling histories. Full details concerning these calibrations and the various uses of HeFTy are publicly available (Carlson and others, 1999; Donelick and others, 1999; Ketcham and others, 1999: Ketcham and others, 2007). For each sample modeled, fission-track ages and track length distributions were calculated for a series of 20,000 randomly generated temperature histories. These randomly generated temperature histories were constrained to be geologically reasonable, where possible, by independently known information such as the intrusion age and present-day geological temperature for each sample. A temperature history was deemed acceptable (i.e., Acceptable Fit; solutions within the green band) when both the model fission-track age and the model fission track length distribution matched their measured counterparts with a level of confidence of 0.05 or greater. A temperature history was deemed good (i.e., Good Fit; solutions within the magenta band) when both the model fission-track age and model fission-track length distribution matched their counterparts with a level of confidence of 0.50 or greater. 

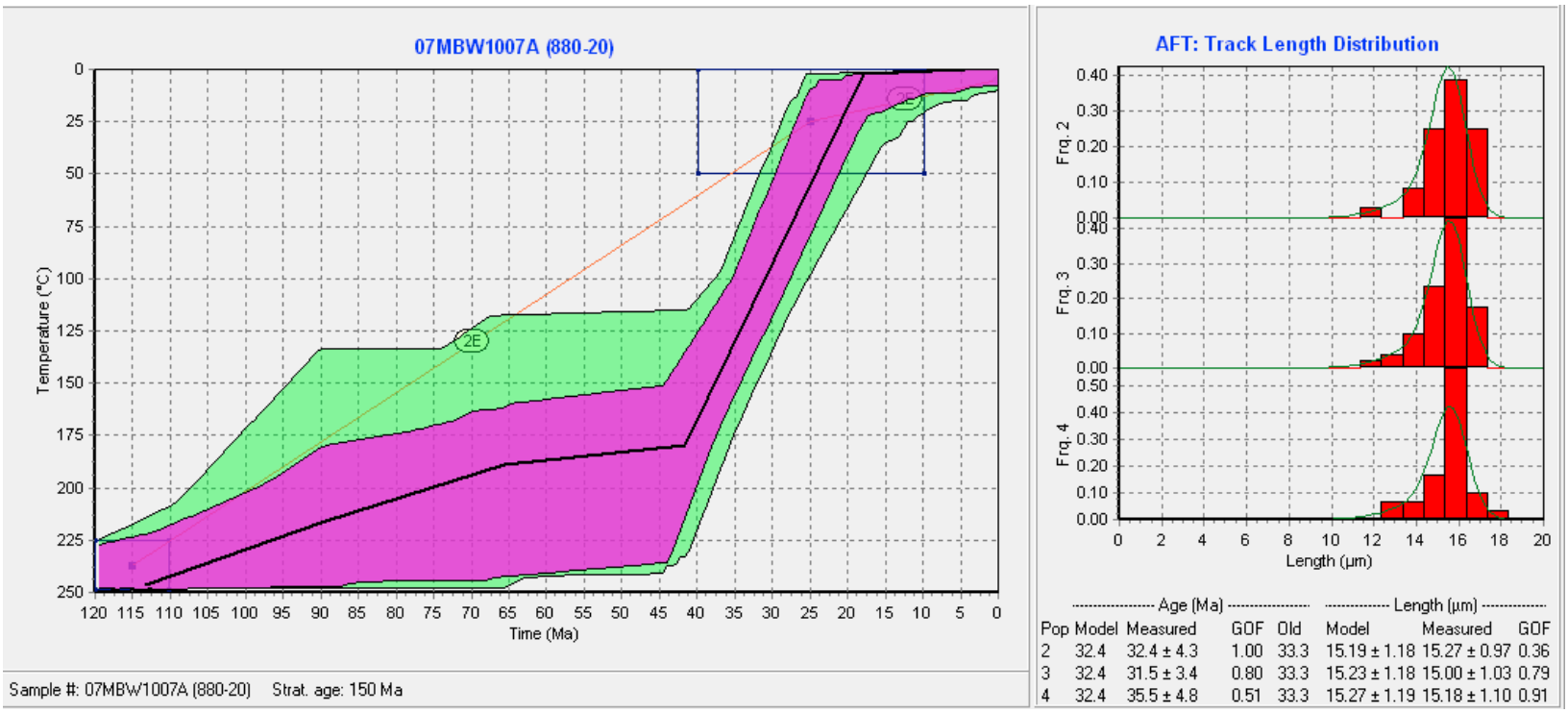

KNOWN PARAMETERS AND ASSUMPTIONS

Client Sample Number

A2Z Sample Number

Kinetic Parameter Modeled

Stratigraphic Age (Ma)

07MBW1007A

880-20

Dpar $(\mu \mathrm{m})$

Jurassic ( 150 Ma)

Present-day Temperature $\left({ }^{\circ} \mathrm{C}\right) \quad 0^{\circ} \mathrm{C}$ Assumed

Timing of Uplift/Cooling (Ma) Assumed to be $<65 \mathrm{Ma}$

\section{IMPLICATIONS OF THE FISSION TRACK DATA}

Age of Oldest Fission Track (Ma) $\quad 33.3 \pm 2.4 \mathrm{Ma}$

(formed during cooling below elevated paleotemperatures)

Timing of Initiation of Uplift/Cooling (Ma) $\geq 33.3 \pm 2.4 \mathrm{Ma}$ 

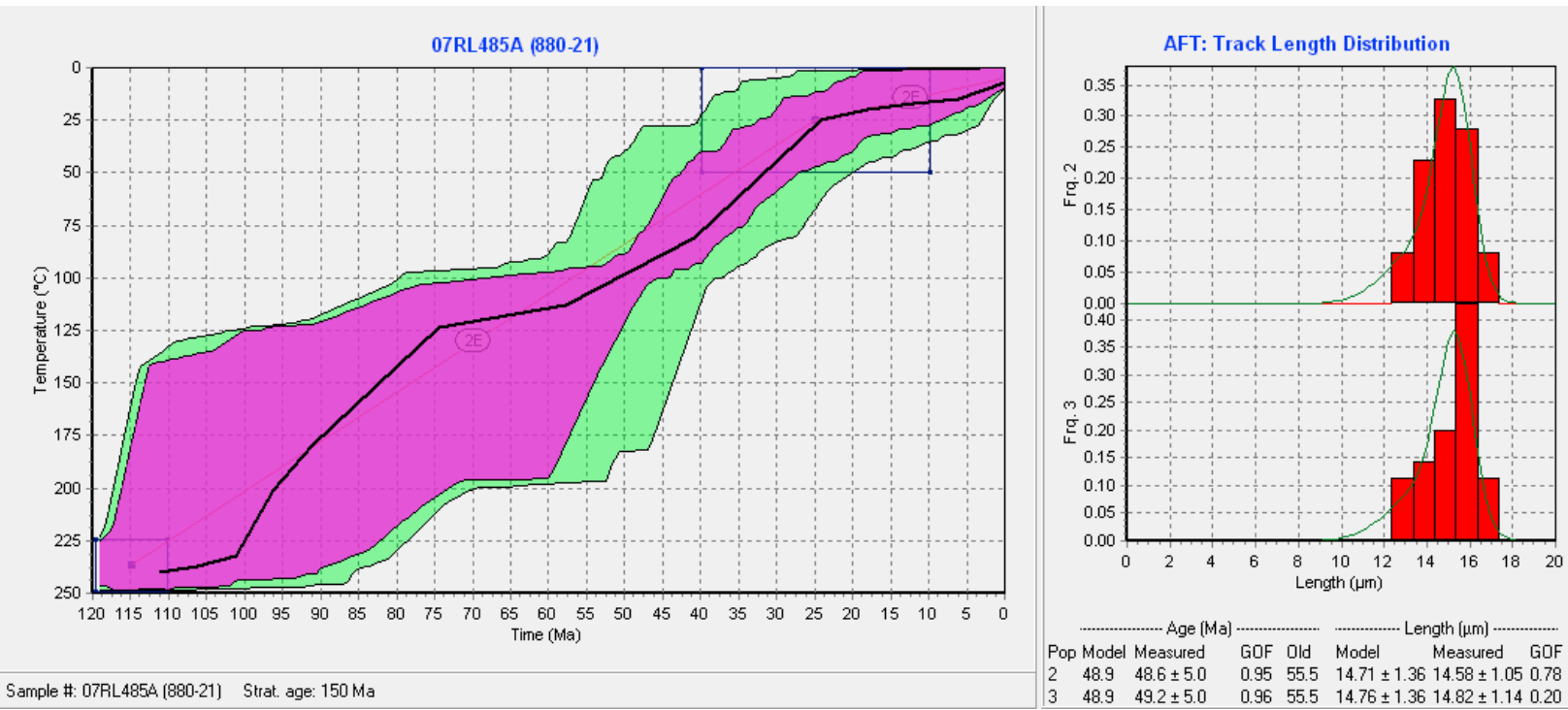

\title{
KNOWN PARAMETERS AND ASSUMPTIONS
}

Client Sample Number

A2Z Sample Number

Kinetic Parameter Modeled

Stratigraphic Age (Ma)

Present-day Temperature $\left({ }^{\circ} \mathrm{C}\right)$

Timing of Uplift/Cooling (Ma)

07RL485A

880-21

Dpar $(\mu \mathrm{m})$

Jurassic ( 150 Ma)

$0^{\circ} \mathrm{C}$ Assumed

Assumed to be $<65 \mathrm{Ma}$

\section{IMPLICATIONS OF THE FISSION TRACK DATA}

\author{
Age of Oldest Fission Track (Ma) $\quad 59.5 \pm 4.4 \mathrm{Ma}$ \\ (formed during cooling below elevated paleotemperatures) \\ Timing of Initiation of Uplift/Cooling (Ma) $\geq 59.5 \pm 4.4 \mathrm{Ma}$
}




\section{BIBLIOGRAPHY}

Burns, L.E., Fugro Airborne Surveys Corp., and Stevens Exploration Management Corp., 2006, Line, grid, and vector data, and plot files for the airborne geophysical survey of the Alaska Highway corridor, east-central Alaska: Fairbanks, Alaska Division of Geological \& Geophysical Surveys Geophysical Report 2006-6, 1 DVD

Burtner, R.L., Nigrini, A., and Donelick, R.A., 1994, Thermochronology of Lower Cretaceous source rocks in the Idaho-Wyoming thrust belt: American Association of Petroleum Geologists Bulletin, v. 78, n. 10, p. 1,6131,636 .

Carver, G.A., Bemis, S.P., Solie, D.N., and Obermiller, K.E., 2008, Active and potentially active faults in or near the Alaska Highway corridor, Delta Junction to Dot Lake, Alaska: Alaska Division of Geological \& Geophysical Surveys Preliminary Interpretive Report 2008-3D, 32 p.

Carlson, W.D., Donelick, R.A., and Ketcham, R.A., 1999, Variability of apatite fission track annealing kinetics I: Experimental results: American Mineralogist, v. 84, p. 1,213-1,223.

Donelick, R.A., Ketcham, R.A., and Carlson, W.D., 1999, Variability of apatite fission track annealing kinetics II: Crystallographic orientation effects: American Mineralogist, v. 84, p. 1,224-1,234.

Donelick, R.A. and Miller, D.S., 1991, Enhanced TINT fission track densities in low spontaneous track density apatites using ${ }^{252} \mathrm{Cf}$-derived fission fragment tracks-A model and experimental observations: Nuclear Tracks and Radiation Measurements, v. 18, p. 301-307.

Donelick, R.A., O’Sullivan, P.B., and Ketcham, R.A., 2005, Apatite fission-track analysis: Reviews in Mineralogy and Geochemistry, v. 58, p. 49-94.

Hasebe, N., Barbarand, J., Jarvis, K., Carter, A., and Hurford, A.J., 2004, Apatite fission-track chronometry using laser ablation ICP-MS: Chemical Geology, v. 207, p. 135-145.

Ketcham, R.A., Donelick, R.A., and Carlson, W.D., 1999, Variability of apatite fission track annealing kinetics III: Extrapolation to geological time scales: American Mineralogist, v. 84, p. 1,235-1,255.

Ketcham, R.A., Carter, A.C., Donelick, R.A., Barbarand, J., and Hurford, A.J., 2007, Improved modeling of fissiontrack annealing in apatite: American Mineralogist, v. 92, p. 799-810.

Werdon, M.B., Solie, D.N., Newberry, R.J., Freeman, L.K., Lessard, R.R., and Elliott, B.A., 2014 in press, Bedrockgeologic map, Alaska Highway corridor, Gerstle River to Dot Lake, Alaska: Alaska Division of Geological \& Geophysical Surveys, Report of Investigations 2014-XX., 1 sheet, scale 1:63,360. 


\section{Appendix A: Apatite FISSION-TRACK Age DAta}

\section{MBW1007A:}

\begin{tabular}{|c|c|c|c|c|c|c|c|c|c|c|c|c|c|c|c|c|c|}
\hline $880-20$ & & & & & & & & & & & & & & & & & \\
\hline & & & & & & & & & & & & & & & & & \\
\hline $\begin{array}{l}\text { Grain } \\
\text { Number }\end{array}$ & Natural & Area & $238 \mathrm{U} / 43 \mathrm{C}$ & 1 sigma & $43 \mathrm{Ca}$ & $238 \mathrm{U}$ & 232Th & 147Sm & FT Age & 1 sigma & $\begin{array}{l}\text { Etch } \\
\text { Figures }\end{array}$ & Dpar & Dper & Uranium & Thorium & Samarium & $\begin{array}{l}\text { Pit Depth } \\
\text { (microns) }\end{array}$ \\
\hline Number & & (cmxcm) & (dmnis) & (dmnls) & back:SIg & back:sig & back:sig & back:sig & (Ma) & & Figures & (microns) & (microns) & (ppm) & (ppm) & (ppm) & (microns) \\
\hline 1 & 12 & $2.91 \mathrm{E}-05$ & $1.37 \mathrm{E}-01$ & $1.12 \mathrm{E}-02$ & $1.62 \mathrm{E}-02$ & $7.08 \mathrm{E}-04$ & $4.76 \mathrm{E}-04$ & 4.94E-04 & 16.27 & 4.89 & 3 & 1.13 & 0.21 & 71.72 & 250.56 & 1106.00 & 11.12 \\
\hline 4 & 3 & 1.16E-05 & $1.92 \mathrm{E}-02$ & $1.86 \mathrm{E}-03$ & $2.00 \mathrm{E}-02$ & $4.28 \mathrm{E}-03$ & 1.44E-02 & 5.17E-03 & 72.51 & 42.48 & 2 & 1.37 & 0.38 & 10.01 & 26.41 & 339.59 & 11.12 \\
\hline 5 & 4 & $1.70 \mathrm{E}-05$ & $1.50 \mathrm{E}-02$ & $2.21 \mathrm{E}-03$ & $2.05 \mathrm{E}-02$ & $2.16 \mathrm{E}-03$ & 7.04E-03 & 2.22E-03 & 84.90 & 44.31 & 1 & 1.34 & 0.31 & 7.81 & 24.37 & 355.92 & 10.47 \\
\hline 6 & 7 & $2.18 \mathrm{E}-05$ & $5.23 \mathrm{E}-02$ & $6.21 \mathrm{E}-03$ & $1.84 \mathrm{E}-02$ & $2.28 \mathrm{E}-04$ & $4.83 \mathrm{E}-03$ & 3.31E-03 & 33.17 & 13.16 & 1 & 1.25 & 0.10 & 27.32 & 63.99 & 429.37 & 11.30 \\
\hline 7 & 1 & 9.71E-06 & $1.94 \mathrm{E}-02$ & $1.80 \mathrm{E}-03$ & $2.09 \mathrm{E}-02$ & $4.02 \mathrm{E}-03$ & 8.67E-03 & 3.12E-03 & 28.77 & 28.90 & 1 & 1.24 & 0.21 & 10.13 & 31.96 & 408.13 & 10.40 \\
\hline & & & & & & & & & & & & & & & & & \\
\hline 8 & 6 & 2.91E-05 & $3.79 \mathrm{E}-02$ & $2.95 \mathrm{E}-03$ & $2.05 \mathrm{E}-02$ & $3.92 \mathrm{E}-03$ & 2.57E-03 & $1.26 \mathrm{E}-03$ & 29.44 & 12.25 & 2 & 1.03 & 0.28 & 19.79 & 46.80 & 438.30 & 10.70 \\
\hline 10 & 10 & $1.46 \mathrm{E}-05$ & $5.94 \mathrm{E}-02$ & $1.11 \mathrm{E}-02$ & $2.10 \mathrm{E}-02$ & $1.24 \mathrm{E}-02$ & $3.84 \mathrm{E}-03$ & 3.38E-03 & 62.42 & 22.96 & 1 & 1.23 & 0.21 & 31.05 & 93.48 & 489.85 & 10.47 \\
\hline 12 & 10 & 1.94E-05 & $1.11 \mathrm{E}-01$ & $8.88 \mathrm{E}-03$ & $2.36 \mathrm{E}-02$ & $9.63 \mathrm{E}-03$ & 8.04E-04 & 8.80E-04 & 25.14 & 8.22 & 2 & 1.48 & 0.39 & 57.97 & 214.37 & 898.02 & 8.67 \\
\hline 13 & 27 & 2.33E-05 & $1.52 \mathrm{E}-01$ & $1.45 \mathrm{E}-02$ & $2.22 \mathrm{E}-02$ & $4.84 \mathrm{E}-04$ & $1.05 \mathrm{E}-03$ & 1.03E-03 & 41.31 & 8.92 & 1 & 1.46 & 0.43 & 79.28 & 229.43 & 1078.10 & 9.66 \\
\hline 14 & 8 & 1.94E-05 & $3.62 \mathrm{E}-02$ & $4.34 \mathrm{E}-03$ & $2.40 \mathrm{E}-02$ & $8.45 \mathrm{E}-03$ & $1.35 \mathrm{E}-02$ & 6.52E-03 & 61.43 & 22.97 & 2 & 1.25 & 0.29 & 18.93 & 44.89 & 428.40 & 9.49 \\
\hline 16 & 6 & $1.21 \mathrm{E}-05$ & $1.35 \mathrm{E}-01$ & $1.32 \mathrm{E}-02$ & $2.50 \mathrm{E}-02$ & $1.77 \mathrm{E}-03$ & $1.20 \mathrm{E}-02$ & $5.08 \mathrm{E}-03$ & 19.80 & 8.32 & 1 & 1.43 & 0.30 & 70.69 & 38.09 & 414.29 & 8.89 \\
\hline 17 & 6 & 9.71E-06 & $2.58 \mathrm{E}-02$ & $3.20 \mathrm{E}-03$ & $4.01 \mathrm{E}-02$ & $1.84 \mathrm{E}-01$ & 2.23E-02 & $6.92 \mathrm{E}-03$ & 128.93 & 55.09 & 2 & 1.44 & 0.41 & 13.46 & 27.93 & 414.79 & 5.76 \\
\hline 18 & 3 & 2.91E-05 & $3.17 \mathrm{E}-02$ & 2.97E-03 & $1.74 \mathrm{E}-02$ & $4.33 \mathrm{E}-02$ & 9.13E-03 & 5.17E-03 & 17.59 & 10.29 & 1 & 1.40 & 0.23 & 16.58 & 42.78 & 348.00 & 12.32 \\
\hline 19 & 3 & 1.16E-05 & $2.83 \mathrm{E}-02$ & $1.62 \mathrm{E}-03$ & $2.18 \mathrm{E}-02$ & $4.13 \mathrm{E}-03$ & 7.73E-03 & $3.20 \mathrm{E}-03$ & 49.13 & 28.52 & 1 & 1.24 & 0.20 & 14.81 & 39.39 & 436.89 & 10.37 \\
\hline & & & & & & & & & & & & & & & & & \\
\hline 20 & 7 & 3.40E-05 & $4.77 \mathrm{E}-02$ & $2.76 \mathrm{E}-03$ & $2.10 \mathrm{E}-02$ & $2.26 \mathrm{E}-03$ & $4.33 \mathrm{E}-03$ & 2.42E-03 & 23.39 & 8.96 & 2 & 1.20 & 0.39 & 24.93 & 54.55 & 448.61 & 10.35 \\
\hline 21 & 2 & 9.71E-06 & $2.34 \mathrm{E}-02$ & $1.71 \mathrm{E}-03$ & $1.92 \mathrm{E}-02$ & $2.38 \mathrm{E}-02$ & 7.81E-03 & $3.11 \mathrm{E}-03$ & 47.53 & 33.80 & 1 & 1.31 & 0.26 & 12.25 & 45.57 & 527.14 & 11.81 \\
\hline 22 & 12 & $1.21 \mathrm{E}-05$ & $8.91 \mathrm{E}-02$ & $9.59 \mathrm{E}-03$ & $2.25 \mathrm{E}-02$ & $8.65 \mathrm{E}-04$ & $2.09 \mathrm{E}-03$ & 1.74E-03 & 59.99 & 18.53 & 1 & 1.18 & 0.21 & 46.52 & 107.80 & 596.69 & 10.52 \\
\hline 23 & 12 & $1.75 \mathrm{E}-05$ & $3.47 \mathrm{E}-02$ & $3.11 \mathrm{E}-03$ & $2.42 \mathrm{E}-02$ & $1.99 \mathrm{E}-03$ & 7.95E-03 & 4.49E-03 & 106.63 & 32.32 & 3 & 1.17 & 0.38 & 18.11 & 47.34 & 384.75 & 10.28 \\
\hline 24 & 16 & 3.11E-05 & $3.20 \mathrm{E}-02$ & $2.34 \mathrm{E}-03$ & $2.58 \mathrm{E}-02$ & $1.73 \mathrm{E}-03$ & $6.40 \mathrm{E}-03$ & 3.96E-03 & 86.72 & 22.67 & 2 & 1.28 & 0.29 & 16.73 & 57.32 & 426.17 & 9.15 \\
\hline 25 & 16 & 4.85E-05 & $4.78 \mathrm{E}-02$ & 4.88E-03 & 2.25E-02 & 4.77E-02 & 6.16E-03 & 4.80E-03 & 37.30 & 10.10 & 2 & 1.43 & 0.34 & 24.99 & 63.92 & 377.22 & 10.51 \\
\hline 26 & 6 & 1.55E-05 & $7.95 \mathrm{E}-02$ & 7.32E-03 & $1.91 \mathrm{E}-02$ & $3.60 \mathrm{E}-03$ & 1.70E-03 & 1.96E-03 & 26.31 & 11.03 & 1 & 1.17 & 0.31 & 41.54 & 143.76 & 572.21 & 12.07 \\
\hline 28 & 0 & 4.37E-05 & $2.25 \mathrm{E}-03$ & $1.91 \mathrm{E}-04$ & $2.35 \mathrm{E}-02$ & $9.19 \mathrm{E}-03$ & $2.30 \mathrm{E}+00$ & 3.31E-01 & 0.00 & 81.86 & 1 & 1.10 & 0.11 & 1.17 & 0.14 & 4.38 & 9.80 \\
\hline 29 & 5 & 2.43E-05 & $2.50 \mathrm{E}-02$ & $2.01 \mathrm{E}-03$ & $2.32 \mathrm{E}-02$ & $0.00 \mathrm{E}+00$ & $5.29 \mathrm{E}-03$ & $2.54 \mathrm{E}-03$ & 44.54 & 20.26 & 1 & 1.79 & 0.27 & 13.07 & 33.88 & 323.49 & 10.25 \\
\hline & & & & & & & & & & & & & & & & & \\
\hline 30 & 5 & 2.43E-05 & $2.51 \mathrm{E}-02$ & 2.34E-03 & 2.10E-02 & 6.86E-04 & $3.36 \mathrm{E}-03$ & 2.49E-03 & 44.33 & 20.27 & 1 & 1.28 & 0.30 & 13.13 & 56.76 & 352.03 & 11.74 \\
\hline 31 & 22 & $2.18 \mathrm{E}-05$ & $1.22 \mathrm{E}-01$ & $9.25 \mathrm{E}-03$ & 2.43E-02 & $3.29 \mathrm{E}-03$ & 3.23E-03 & 3.35E-03 & 44.70 & 10.16 & 2 & 1.40 & 0.29 & 63.67 & 183.62 & 815.40 & 9.88 \\
\hline 33 & 5 & 2.43E-05 & $2.21 \mathrm{E}-02$ & $2.26 \mathrm{E}-03$ & 2.43E-02 & $6.42 \mathrm{E}-03$ & $6.80 \mathrm{E}-03$ & $2.25 \mathrm{E}-03$ & 50.40 & 23.15 & 1 & 1.19 & 0.18 & 11.55 & 25.60 & 354.97 & 9.76 \\
\hline 34 & 7 & 1.94E-05 & $4.15 \mathrm{E}-01$ & $5.48 \mathrm{E}-02$ & 2.61E-02 & $3.04 \mathrm{E}-03$ & 8.56E-04 & 1.40E-03 & 4.72 & 1.89 & 1 & 1.25 & 0.31 & 216.64 & 399.66 & 1124.90 & 9.51 \\
\hline 35 & 3 & 1.94E-05 & $2.37 \mathrm{E}-02$ & 2.26E-03 & 2.40E-02 & 5.77E-03 & $1.22 \mathrm{E}-02$ & 4.64E-03 & 35.31 & 20.68 & 1 & 1.40 & 0.27 & 12.37 & 28.83 & 348.10 & 10.14 \\
\hline 37 & 8 & 1.75E-05 & $3.61 \mathrm{E}-02$ & 2.59E-03 & 2.58E-02 & 2.35E-03 & 1.19E-02 & 5.59E-03 & 68.42 & 24.73 & 1 & 1.34 & 0.20 & 18.87 & 41.59 & 407.23 & 9.53 \\
\hline 38 & 8 & $1.94 \mathrm{E}-05$ & $3.40 \mathrm{E}-02$ & 2.66E-03 & 2.37E-02 & 2.32E-03 & 5.06E-03 & 2.63E-03 & 65.38 & 23.71 & 2 & 1.33 & 0.42 & 17.78 & 50.20 & 444.99 & 10.28 \\
\hline 39 & 9 & 1.36E-05 & $1.13 \mathrm{E}-01$ & 1.06E-02 & 2.25E-02 & 1.09E-03 & 1.15E-03 & $1.16 \mathrm{E}-03$ & 31.68 & 10.99 & 1 & 1.24 & 0.21 & 59.13 & 178.89 & 814.47 & 10.42 \\
\hline 40 & 2 & 9.71E-06 & $9.69 \mathrm{E}-03$ & $1.71 \mathrm{E}-03$ & 3.55E-02 & $1.28 \mathrm{E}-02$ & $2.86 \mathrm{E}-02$ & 7.01E-02 & 114.43 & 83.44 & 1 & 1.10 & 0.26 & 5.06 & 27.88 & 52.31 & 7.10 \\
\hline & & & & & & & & & & & & & & & & & \\
\hline & & & & & & & & & & & & & & & & & \\
\hline & Mean & Dpar & $=$ & 1.29 & & & & & & & & & & & & & \\
\hline & Mean & Dper & $=$ & 0.28 & & & & & & & & & & & & & \\
\hline & Modified & Zeta & $=$ & $10.856+/-$ & 0.238 & & & & & & & & & & & & \\
\hline & Mean & $29 \mathrm{Si}$ & b:s & $=$ & 0.023 & & & & & & & & & & & & \\
\hline & Mean & $43 \mathrm{Ca}$ & b:s & $=$ & 0.023 & & & & & & & & & & & & \\
\hline & Mean & $238 \mathrm{U}$ & b:s & $=$ & 0.013 & & & & & & & & & & & & \\
\hline & Number & of & grains= & 32 & & & & & & & & & & & & & \\
\hline & Chi-square $=$ & $=$ & 104.6 & & & & & & & & & & & & & & \\
\hline & Chi-square & prob $=$ & 0.0000 & & & & & & & & & & & & & & \\
\hline & Pooled & Age & (Ma) & $=$ & $32.9+/-$ & 2.4 & & & & & & & & & & & \\
\hline
\end{tabular}


07RL485A:

880-21

\begin{tabular}{|l|l|l|l|l|l|l|l|l|l|l|l|l|l}
\hline Grain Natural & Area & $238 \mathrm{U} / 43 \mathrm{C}$ & 1 sigma & $43 \mathrm{Ca}$ & $238 \mathrm{U}$ & $232 \mathrm{Th}$ & $147 \mathrm{Sm}$ & FT Age & 1 sigma & Etch & Dpar & Dper Uranium Thorium Samarium Pit Depth
\end{tabular} Number Tracks (cmxcm) (dmnls) (dmnls) back:sig back:sig back:sig back:sig (Ma) $\quad$ (Ma) Figures $\quad$ (microns) (microns) (ppm) (ppm) (ppm) (microns)

\begin{tabular}{|c|c|c|c|c|c|c|c|c|c|c|c|c|c|c|c|c|c|}
\hline 1 & 15 & $2.33 \mathrm{E}-05$ & 5.93E-02 & 4.49E-03 & $1.91 \mathrm{E}-02$ & $4.52 \mathrm{E}-04$ & $2.71 \mathrm{E}-03$ & $1.70 \mathrm{E}-03$ & 57.44 & 15.51 & 2 & 1.09 & 0.32 & 31.64 & 112.55 & 825.32 & 11.52 \\
\hline 2 & 8 & $2.91 \mathrm{E}-05$ & $3.22 \mathrm{E}-02$ & $1.85 \mathrm{E}-03$ & $2.10 \mathrm{E}-02$ & $6.15 \mathrm{E}-02$ & $2.37 \mathrm{E}-02$ & $7.85 \mathrm{E}-03$ & 45.21 & 16.22 & 2 & 1.18 & 0.16 & 17.17 & 45.75 & 636.74 & 10.79 \\
\hline 3 & 17 & $2.04 \mathrm{E}-05$ & $4.31 \mathrm{E}-02$ & $2.56 \mathrm{E}-03$ & $2.55 \mathrm{E}-02$ & $3.02 \mathrm{E}-02$ & $2.20 \mathrm{E}-02$ & $9.39 \mathrm{E}-03$ & 102.03 & 25.57 & 1 & 1.37 & 0.22 & 22.99 & 65.36 & 706.50 & 10.23 \\
\hline 4 & 8 & $2.38 \mathrm{E}-05$ & 2.49E-02 & $1.53 \mathrm{E}-03$ & $2.59 \mathrm{E}-02$ & $3.42 \mathrm{E}-03$ & $2.42 \mathrm{E}-02$ & $5.82 \mathrm{E}-03$ & 71.32 & 25.64 & 1 & 1.16 & 0.41 & 13.30 & 29.24 & 559.76 & 9.64 \\
\hline 5 & 21 & $1.94 \mathrm{E}-05$ & $1.18 \mathrm{E}-01$ & $1.53 \mathrm{E}-02$ & $3.42 \mathrm{E}-02$ & $2.77 \mathrm{E}-03$ & $1.53 \mathrm{E}-03$ & $1.40 \mathrm{E}-03$ & 48.39 & 12.32 & 1 & 1.25 & 0.37 & 63.14 & 308.37 & 1555.50 & 6.71 \\
\hline 6 & 9 & $2.43 \mathrm{E}-05$ & $3.46 \mathrm{E}-02$ & 2.93E-03 & 2.62E-02 & $8.87 \mathrm{E}-04$ & 3.30E-03 & $1.30 \mathrm{E}-03$ & 56.70 & 19.54 & 1 & 1.28 & 0.24 & 18.46 & 59.62 & 694.35 & 8.93 \\
\hline 7 & 7 & $1.75 \mathrm{E}-05$ & $5.72 \mathrm{E}-02$ & $5.36 \mathrm{E}-03$ & 4.04E-02 & $3.85 \mathrm{E}-02$ & $1.23 \mathrm{E}-02$ & $1.03 \mathrm{E}-02$ & 37.10 & 14.47 & 1 & 1.45 & 0.43 & 30.53 & 175.42 & 963.90 & 5.94 \\
\hline 8 & 17 & $2.33 \mathrm{E}-05$ & $6.45 \mathrm{E}-02$ & 3.33E-03 & $2.52 \mathrm{E}-02$ & $7.15 \mathrm{E}-03$ & $2.40 \mathrm{E}-03$ & 2.03E-03 & 59.84 & 14.90 & 2 & 1.46 & 0.33 & 34.42 & 151.19 & 821.93 & 9.90 \\
\hline 10 & 11 & $3.88 \mathrm{E}-05$ & 2.47E-02 & $1.28 \mathrm{E}-03$ & $2.52 \mathrm{E}-02$ & $4.33 \mathrm{E}-02$ & $2.56 \mathrm{E}-02$ & 1.07E-02 & 60.57 & 18.58 & 2 & 1.34 & 0.38 & 13.20 & 49.57 & 548.79 & 9.52 \\
\hline 11 & 4 & $1.16 \mathrm{E}-05$ & 4.64E-02 & 3.17E-03 & $2.14 \mathrm{E}-02$ & $9.13 \mathrm{E}-03$ & $6.61 \mathrm{E}-03$ & $2.76 \mathrm{E}-03$ & 39.18 & 19.79 & 2 & 1.36 & 0.19 & 24.77 & 73.45 & 808.84 & 10.85 \\
\hline 12 & 14 & $2.91 \mathrm{E}-05$ & $6.67 \mathrm{E}-02$ & $6.70 \mathrm{E}-03$ & $2.29 \mathrm{E}-02$ & $1.78 \mathrm{E}-02$ & $3.29 \mathrm{E}-03$ & $1.87 \mathrm{E}-03$ & 38.17 & 10.93 & 2 & 1.35 & 0.30 & 35.60 & 109.12 & 883.84 & 10.60 \\
\hline 13 & 15 & $2.04 \mathrm{E}-05$ & $8.26 \mathrm{E}-02$ & 7.27E-03 & $2.42 \mathrm{E}-02$ & $8.16 \mathrm{E}-02$ & $2.83 \mathrm{E}-03$ & 2.00E-03 & 47.19 & 12.92 & 1 & 1.11 & 0.26 & 44.05 & 159.31 & 1035.10 & 9.46 \\
\hline 14 & 16 & $1.70 \mathrm{E}-05$ & 7.07E-02 & 6.65E-03 & 2.23E-02 & 1.30E-01 & 4.92E-03 & 3.36E-03 & 70.39 & 18.87 & 1 & 1.40 & 0.21 & 37.73 & 135.73 & 916.21 & 10.36 \\
\hline 15 & 4 & $1.21 \mathrm{E}-05$ & 6.69E-02 & 7.48E-03 & $2.28 \mathrm{E}-02$ & $0.00 E+00$ & $2.01 \mathrm{E}-03$ & $1.14 \mathrm{E}-03$ & 26.13 & 13.40 & 2 & 1.12 & 0.28 & 35.70 & 107.45 & 870.11 & 10.25 \\
\hline 16 & 8 & $2.91 \mathrm{E}-05$ & $2.60 \mathrm{E}-02$ & $3.22 \mathrm{E}-03$ & 2.00E-02 & $1.80 \mathrm{E}-01$ & $2.74 \mathrm{E}-02$ & $1.10 \mathrm{E}-02$ & 55.94 & 20.99 & 1 & 1.03 & 0.21 & 13.86 & 50.93 & 581.68 & 11.44 \\
\hline 18 & 1 & $9.71 \mathrm{E}-06$ & $3.29 \mathrm{E}-02$ & $4.02 \mathrm{E}-03$ & $2.46 \mathrm{E}-02$ & $3.23 \mathrm{E}-03$ & $4.73 \mathrm{E}-03$ & $1.98 \mathrm{E}-03$ & 16.64 & 16.77 & 1 & 1.34 & 0.23 & 17.53 & 66.80 & 734.56 & 10.34 \\
\hline 19 & 6 & $4.37 \mathrm{E}-06$ & 4.76E-02 & 8.11E-03 & $2.18 \mathrm{E}-02$ & $2.72 \mathrm{E}-01$ & $2.26 \mathrm{E}-02$ & $2.08 \mathrm{E}-02$ & 151.62 & 67.15 & 2 & 1.32 & 0.22 & 25.38 & 139.24 & 697.67 & 11.36 \\
\hline 20 & 8 & $2.91 \mathrm{E}-05$ & 4.23E-02 & 8.44E-03 & 2.71E-01 & $6.14 \mathrm{E}-01$ & 8.47E-01 & $3.11 \mathrm{E}-01$ & 34.41 & 13.99 & 1 & 1.50 & 0.41 & 22.57 & 65.20 & 816.50 & 9.89 \\
\hline 21 & 20 & $3.11 \mathrm{E}-05$ & 4.54E-02 & $5.43 \mathrm{E}-03$ & $2.30 \mathrm{E}-02$ & 2.91E-03 & $4.08 \mathrm{E}-03$ & $1.98 \mathrm{E}-03$ & 74.97 & 19.08 & 0 & 0.00 & 0.00 & 24.21 & 82.22 & 777.85 & 10.90 \\
\hline 22 & 12 & $2.43 \mathrm{E}-05$ & $2.85 \mathrm{E}-02$ & 3.56E-03 & 2.23E-02 & $8.78 \mathrm{E}-04$ & $7.80 \mathrm{E}-03$ & $2.92 \mathrm{E}-03$ & 91.69 & 28.92 & 1 & 1.18 & 0.25 & 15.18 & 46.82 & 574.99 & 10.97 \\
\hline 23 & 28 & $2.91 \mathrm{E}-05$ & $1.41 \mathrm{E}-01$ & 1.81E-02 & $2.29 \mathrm{E}-02$ & $3.97 \mathrm{E}-02$ & $2.75 \mathrm{E}-03$ & $2.40 \mathrm{E}-03$ & 36.18 & 8.31 & 1 & 1.39 & 0.41 & 75.13 & 257.24 & 1354.80 & 11.25 \\
\hline 24 & 13 & $2.43 \mathrm{E}-05$ & $9.57 \mathrm{E}-02$ & 9.59E-03 & $2.50 \mathrm{E}-02$ & $5.92 \mathrm{E}-04$ & $1.58 \mathrm{E}-03$ & $1.18 \mathrm{E}-03$ & 29.69 & 8.78 & 3 & 1.22 & 0.34 & 51.05 & 163.06 & 1002.60 & 9.68 \\
\hline 25 & 7 & $1.94 \mathrm{E}-05$ & $6.77 \mathrm{E}-02$ & $9.48 \mathrm{E}-03$ & $1.70 \mathrm{E}-02$ & $4.40 \mathrm{E}-02$ & $2.75 \mathrm{E}-02$ & $2.22 \mathrm{E}-02$ & 28.26 & 11.41 & 0 & 0.00 & 0.00 & 36.09 & 151.52 & 863.64 & 14.26 \\
\hline & & & & & & & & & & & & & & & & & \\
\hline & Mean & Dpar & $=$ & 1.28 & & & & & & & & & & & & & \\
\hline & Mean & Dper & $=$ & 0.29 & & & & & & & & & & & & & \\
\hline & Modified & Zeta & $=$ & $10.631+/-$ & 0.233 & & & & & & & & & & & & \\
\hline & Mean & $29 \mathrm{Si}$ & b:s & $=$ & 0.035 & & & & & & & & & & & & \\
\hline & Mean & $43 \mathrm{Ca}$ & b:s & $=$ & 0.035 & & & & & & & & & & & & \\
\hline & Mean & $238 \mathrm{U}$ & b:s & $=$ & 0.069 & & & & & & & & & & & & \\
\hline & Number & of & grains= & 23 & & & & & & & & & & & & & \\
\hline & Chi-square & & 38.3 & & & & & & & & & & & & & & \\
\hline & Chi-square & e prob= & 0.0170 & & & & & & & & & & & & & & \\
\hline & Pooled & Age & (Ma) & $=$ & $49.2+/-$ & 3.5 & & & & & & & & & & & \\
\hline
\end{tabular}




\section{Appendix B: Apatite Fission-Track LENGTh DATA}

\section{MBW1007A}

\section{0-20}

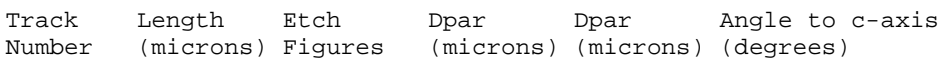

\begin{tabular}{|c|c|c|c|c|c|}
\hline 1 & 14.41 & 2 & 1.28 & 0.11 & 79.21 \\
\hline 2 & 12.23 & 2 & 1.11 & 0.02 & 56.66 \\
\hline 3 & 15.76 & 2 & 1.05 & 0.21 & 63.41 \\
\hline 4 & 11.02 & 2 & 1.15 & 0.20 & $90.0 \odot$ \\
\hline 5 & 14.31 & 2 & 1.15 & 0.20 & 33.96 \\
\hline 6 & 13.21 & 4 & 1.11 & 0.09 & 62.50 \\
\hline 7 & 15.33 & 1 & 1.19 & 0.18 & 48.23 \\
\hline 8 & 14.65 & 1 & 1.37 & 0.14 & 76.12 \\
\hline 9 & 15.45 & 2 & 1.11 & 0.18 & 13.70 \\
\hline 10 & 15.99 & 2 & 1.37 & 0.15 & 40.86 \\
\hline 11 & 14.39 & 2 & 1.37 & 0.15 & 49.45 \\
\hline 12 & 14.07 & 2 & 1.37 & 0.15 & 31.48 \\
\hline 13 & 13.22 & 2 & 1.12 & 0.24 & 60.08 \\
\hline 14 & 13.05 & 2 & 1.12 & 0.24 & 66.19 \\
\hline 15 & 13.84 & 2 & 0.90 & 0.12 & 84.36 \\
\hline 16 & 14.63 & 2 & 0.90 & 0.12 & 45.95 \\
\hline 17 & 15.40 & 2 & 1.15 & 0.16 & 47.79 \\
\hline 18 & 13.90 & 1 & 1.40 & 0.25 & 63.52 \\
\hline 19 & 13.92 & 2 & 1.38 & 0.25 & 56.85 \\
\hline 20 & 12.48 & 2 & 1.38 & 0.25 & 72.98 \\
\hline 21 & 14.52 & 2 & 1.34 & 0.10 & 60.89 \\
\hline 22 & 14.88 & 2 & 1.34 & 0.10 & 31.47 \\
\hline 23 & 15.88 & 2 & 1.52 & 0.16 & 57.96 \\
\hline 24 & 15.20 & 2 & 1.52 & 0.16 & 40.42 \\
\hline 25 & 9.01 & 2 & 1.52 & 0.16 & 83.87 \\
\hline 26 & 16.91 & 2 & 1.52 & 0.16 & 58.15 \\
\hline 27 & 16.68 & 2 & 1.52 & 0.16 & 54.46 \\
\hline 28 & 14.84 & 2 & 1.52 & 0.16 & 79.32 \\
\hline 29 & 14.55 & 2 & 1.12 & $-\odot . \odot 2$ & 61.20 \\
\hline 30 & 16.14 & 2 & 1.12 & $-\odot . \odot 2$ & 63.97 \\
\hline 31 & 15.37 & 2 & 1.12 & $-\odot . \odot 2$ & 28.40 \\
\hline 32 & 13.74 & 2 & 1.19 & 0.21 & 54.47 \\
\hline 33 & 11.69 & 2 & 1.15 & $-\odot . \odot \odot$ & 68.44 \\
\hline 34 & 15.07 & 2 & 1.19 & 0.21 & 62.95 \\
\hline 35 & 12.39 & 1 & 1.25 & 0.11 & 62.63 \\
\hline 36 & 14.71 & 1 & 1.25 & 0.11 & 33.62 \\
\hline 37 & 13.06 & 3 & 1.23 & 0.30 & 73.49 \\
\hline 38 & 13.32 & 3 & 1.23 & 0.30 & 55.71 \\
\hline 39 & 13.03 & 3 & 1.23 & 0.30 & 73.20 \\
\hline 40 & 15.42 & 3 & 1.23 & 0.30 & 35.18 \\
\hline 41 & 15.37 & 4 & 1.71 & 0.25 & 29.49 \\
\hline 42 & 13.72 & 4 & 1.19 & 0.12 & 60.33 \\
\hline 43 & 4.80 & 4 & 1.19 & 0.12 & 74.70 \\
\hline 44 & 15.69 & 4 & 1.19 & 0.12 & 74.67 \\
\hline 45 & 13.74 & 4 & 1.33 & 0.20 & 72.06 \\
\hline 46 & 16.30 & 4 & 1.33 & 0.20 & 9.11 \\
\hline 47 & 11.56 & 2 & 1.37 & 0.23 & 84.85 \\
\hline 48 & 12.83 & 2 & 1.37 & 0.23 & 33.46 \\
\hline 49 & 14.63 & 2 & 1.37 & 0.23 & 36.34 \\
\hline 50 & 12.77 & 2 & 1.37 & 0.23 & 73.44 \\
\hline 51 & 14.81 & 2 & 1.37 & 0.23 & 52.09 \\
\hline 52 & 15.55 & 2 & 1.32 & 0.38 & 56.24 \\
\hline 53 & 15.38 & 2 & 1.32 & 0.38 & 23.35 \\
\hline 54 & 15.81 & 2 & 1.32 & 0.38 & 36.46 \\
\hline 55 & 15.88 & 2 & 1.32 & 0.38 & 34.56 \\
\hline 56 & 15.40 & 2 & 1.48 & 0.12 & 50.23 \\
\hline 57 & 16.02 & 2 & 1.48 & 0.12 & 45.17 \\
\hline 58 & 13.09 & 2 & 1.48 & 0.12 & 85.26 \\
\hline 59 & 15.97 & 2 & 1.48 & 0.12 & 10.30 \\
\hline 60 & 14.45 & 3 & 1.38 & 0.40 & 52.52 \\
\hline 61 & 15.52 & 3 & 1.38 & 0.40 & 36.99 \\
\hline 62 & 16.03 & 2 & 1.39 & 0.19 & 70.12 \\
\hline 63 & 11.32 & 1 & 1.50 & 0.25 & 60.43 \\
\hline 64 & 13.73 & 1 & 1.50 & 0.25 & 37.76 \\
\hline 65 & 15.32 & 1 & 1.50 & 0.25 & 34.22 \\
\hline 66 & 14.08 & 1 & 1.57 & -0.03 & 52.20 \\
\hline 67 & 14.57 & 1 & 1.57 & -0.03 & 89.75 \\
\hline 68 & 14.61 & 1 & 1.57 & -0.03 & 51.01 \\
\hline 69 & 14.17 & 2 & 1.06 & 0.18 & 79.34 \\
\hline 70 & 11.15 & 3 & 1.34 & 0.15 & 82.63 \\
\hline 71 & 15.01 & 3 & 1.34 & 0.15 & 51.72 \\
\hline
\end{tabular}




\begin{tabular}{|c|c|c|c|c|c|}
\hline 72 & 15.31 & 1 & 1.76 & 0.39 & 34.08 \\
\hline 73 & 15.61 & 3 & 1.14 & 0.21 & 52.65 \\
\hline 74 & 13.48 & 3 & 1.14 & 0.21 & 60.39 \\
\hline 75 & 14.39 & 3 & 1.14 & 0.21 & 57.30 \\
\hline 76 & 15.37 & 3 & 1.14 & $\odot .21$ & 85.97 \\
\hline 77 & 15.76 & 3 & 1.14 & 0.21 & 0.37 \\
\hline 78 & 14.27 & 3 & 1.14 & 0.21 & 50.74 \\
\hline 79 & 15.11 & 2 & 0.87 & 0.51 & 67.61 \\
\hline 80 & 13.52 & 3 & 1.00 & 0.18 & 84.62 \\
\hline 81 & 15.89 & 3 & 1.00 & 0.18 & 32.14 \\
\hline 82 & 14.90 & 3 & 1.18 & 0.21 & 77.31 \\
\hline 83 & 13.35 & 3 & 1.18 & 0.21 & 82.05 \\
\hline 84 & 15.94 & 3 & 1.18 & 0.21 & 51.56 \\
\hline 85 & 15.43 & 3 & 1.18 & $\odot .21$ & 41.33 \\
\hline 86 & 14.64 & 2 & 1.27 & 0.16 & 62.77 \\
\hline 87 & 15.30 & 2 & 1.27 & 0.16 & 24.74 \\
\hline 88 & 13.34 & 2 & 1.27 & 0.16 & 56.70 \\
\hline 89 & 13.72 & 2 & 1.53 & 0.20 & 75.13 \\
\hline 90 & 14.92 & 2 & 1.10 & 0.15 & 50.64 \\
\hline 91 & 14.83 & 3 & 1.26 & 0.25 & 30.63 \\
\hline 92 & 14.32 & 2 & 1.34 & 0.33 & 33.23 \\
\hline 93 & 13.01 & 2 & 1.34 & 0.33 & 50.34 \\
\hline 94 & 14.18 & 4 & 1.53 & 0.21 & 63.07 \\
\hline 95 & 15.65 & 4 & 1.53 & 0.21 & 22.29 \\
\hline 96 & 15.53 & 4 & 1.53 & 0.21 & 39.46 \\
\hline 97 & 15.10 & 4 & 1.45 & 0.28 & 51.38 \\
\hline 98 & 14.17 & 4 & 1.45 & 0.28 & 38.23 \\
\hline 99 & 14.36 & 4 & 1.45 & 0.28 & 83.29 \\
\hline 100 & 13.61 & 4 & 1.45 & 0.28 & 37.63 \\
\hline 101 & 14.85 & 4 & 1.45 & 0.28 & 52.05 \\
\hline 102 & 11.12 & 3 & 1.39 & 0.21 & 41.28 \\
\hline 103 & 11.69 & 3 & 1.39 & 0.21 & 77.48 \\
\hline 104 & 14.00 & 3 & 1.39 & 0.21 & 67.72 \\
\hline 105 & 13.90 & 3 & 1.28 & 0.25 & 77.05 \\
\hline 106 & 15.90 & 3 & 1.19 & 0.34 & 42.99 \\
\hline 107 & 15.34 & 3 & 1.19 & 0.34 & 23.49 \\
\hline 108 & 15.72 & 3 & 1.19 & 0.34 & 51.81 \\
\hline 109 & 16.07 & 3 & 1.19 & 0.34 & 51.77 \\
\hline 110 & 13.98 & 3 & 1.28 & 0.11 & 84.33 \\
\hline 111 & 15.56 & 3 & 1.28 & 0.11 & 49.41 \\
\hline 112 & 13.80 & 4 & 1.25 & 0.14 & 65.34 \\
\hline 113 & 11.44 & 4 & 1.25 & 0.14 & 89.45 \\
\hline 114 & 8.69 & 4 & 1.25 & 0.14 & $59 . \odot \odot$ \\
\hline 115 & 15.83 & 4 & 1.25 & 0.14 & 25.65 \\
\hline 116 & 9.76 & 4 & 1.25 & 0.14 & 69.61 \\
\hline 117 & 16.48 & 4 & 1.25 & 0.14 & 50.57 \\
\hline 118 & 15.84 & 2 & 1.30 & 0.39 & 57.28 \\
\hline 119 & 12.87 & 2 & 1.46 & $\odot .09$ & 78.66 \\
\hline 120 & 10.48 & 2 & 0.93 & 0.04 & 72.18 \\
\hline 121 & 9.86 & 2 & 1.44 & 0.21 & 52.53 \\
\hline 122 & 11.63 & 2 & 1.44 & 0.21 & 68.80 \\
\hline 123 & 14.19 & 2 & 1.44 & 0.21 & 48.77 \\
\hline 124 & 15.75 & 2 & 1.44 & 0.21 & 17.83 \\
\hline 125 & 13.36 & 2 & 1.07 & 0.05 & 71.39 \\
\hline Mean & Dpar & $=1.30$ & & & \\
\hline Mean & Dper & $=0.20$ & & & \\
\hline \multicolumn{6}{|c|}{ Mean length $(u m)=14.16+/-0.16$} \\
\hline \multicolumn{6}{|c|}{$\begin{array}{ll}\text { Std. Dev. } \quad(u m)=1.83\end{array}$} \\
\hline Skew & hess & $=-1.77$ & & & \\
\hline Kurt & osis & $=4.92$ & & & \\
\hline
\end{tabular}


07RL485A

880-21

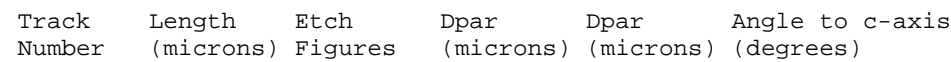

\begin{tabular}{|c|c|c|c|c|c|}
\hline 1 & 13.29 & 2 & 1.19 & 0.07 & 69.45 \\
\hline 2 & 13.85 & 2 & 1.19 & 0.07 & 35.98 \\
\hline 3 & 13.03 & 2 & 1.17 & 0.29 & 44.20 \\
\hline 4 & 14.71 & 2 & 1.40 & 0.02 & 56.02 \\
\hline 5 & 12.17 & 2 & 1.40 & 0.02 & 36.75 \\
\hline 6 & 15.53 & 2 & 1.40 & 0.02 & 27.12 \\
\hline 7 & 13.42 & 3 & 1.27 & 0.05 & 36.27 \\
\hline 8 & 14.29 & 2 & 1.05 & 0.21 & 37.47 \\
\hline 9 & 14.57 & 2 & 1. 27 & 0.21 & 35.72 \\
\hline 10 & 15.29 & 2 & 1.27 & 0.21 & 25.42 \\
\hline 11 & 13.81 & 2 & 1.25 & 0.14 & 76.80 \\
\hline 12 & 12.74 & 2 & 1.05 & 0.31 & 72.06 \\
\hline 13 & 15.34 & 4 & 1.06 & 0.15 & 56.49 \\
\hline 14 & 14.32 & 4 & 1.06 & 0.15 & 34.53 \\
\hline 15 & 15.97 & 2 & 1.30 & 0.20 & 45.12 \\
\hline 16 & 14.74 & 3 & 1.40 & 0.23 & $78.0 \odot$ \\
\hline 17 & 16.07 & 3 & 1.40 & 0.23 & 18.22 \\
\hline 18 & 13.05 & 2 & 1.15 & 0.09 & 75.66 \\
\hline 19 & 12.13 & 3 & 1.28 & 0.20 & 66.78 \\
\hline 20 & 15.80 & 3 & 1.28 & 0.20 & 39.79 \\
\hline 21 & 15.71 & 3 & 1.28 & 0.20 & 32.80 \\
\hline 22 & 16.11 & 3 & 1.28 & 0.20 & 15.41 \\
\hline 23 & 15.03 & 3 & 1.45 & 0.23 & 22.47 \\
\hline 24 & 14.86 & 3 & 1.45 & 0.23 & 53.91 \\
\hline 25 & 13.60 & 3 & 1.15 & 0.24 & 81.04 \\
\hline 26 & 16.42 & 3 & 1.15 & 0.24 & 25.79 \\
\hline 27 & 9.65 & 3 & 1.15 & 0.24 & 77.96 \\
\hline 28 & 15.39 & 3 & 1.15 & 0.24 & 51.93 \\
\hline 29 & 12.88 & 3 & 1. 21 & 0.12 & 64.94 \\
\hline 30 & 14.52 & 3 & 1.21 & 0.12 & 12.20 \\
\hline 31 & 15.41 & 3 & 1.21 & 0.12 & 68.68 \\
\hline 32 & 16.37 & 2 & 1.33 & 0.14 & 39.09 \\
\hline 33 & 12.60 & 4 & 1.30 & 0.12 & 31.73 \\
\hline 34 & 15.57 & 4 & 1.30 & 0.12 & 40.11 \\
\hline 35 & 15.90 & 2 & 1.54 & 0.28 & 42.34 \\
\hline 36 & 13.49 & 2 & 1.54 & 0.28 & 62.88 \\
\hline 37 & 14.77 & 2 & 1.54 & 0.28 & 25.28 \\
\hline 38 & 11.87 & 2 & 1.46 & 0.10 & 63.34 \\
\hline 39 & 11.03 & 2 & 1.27 & 0.25 & 71.97 \\
\hline 40 & 7.49 & 2 & 1. 27 & 0.25 & 89.05 \\
\hline 41 & 11.42 & 2 & 0.93 & 0.20 & 85.08 \\
\hline 42 & 11.98 & 2 & 1.19 & 0.10 & 49.45 \\
\hline 43 & 12.10 & 2 & 1.19 & 0.10 & 49.78 \\
\hline 44 & 13.83 & 1 & 1.19 & 0.16 & 35.14 \\
\hline 45 & 13.24 & 1 & 1.19 & 0.16 & 63.75 \\
\hline 46 & 12.49 & 2 & 1.33 & 0.23 & 66.74 \\
\hline 47 & 14.04 & 2 & 1.17 & 0.19 & 55.41 \\
\hline 48 & 11.02 & 2 & 1.17 & 0.19 & 56.64 \\
\hline 49 & 12.74 & 2 & 1.17 & 0.19 & 70.79 \\
\hline 50 & 10.74 & 3 & 1.07 & 0.20 & 58.19 \\
\hline 51 & 14.39 & 3 & 1.07 & 0.20 & 28.47 \\
\hline 52 & 13.51 & 2 & 1.46 & 0.19 & 51.62 \\
\hline 53 & 14.69 & 2 & 1.46 & 0.19 & 72.54 \\
\hline 54 & 13.60 & 2 & 1.46 & 0.19 & 56.49 \\
\hline 55 & 13.75 & 2 & 1.46 & 0.19 & 67.95 \\
\hline 56 & 14.91 & 2 & 1.01 & 0.11 & 59.68 \\
\hline 57 & 14.09 & 2 & 1.01 & 0.11 & 31.86 \\
\hline 58 & 10.76 & 2 & 1.01 & 0.11 & 72.27 \\
\hline 59 & 11.32 & 2 & 1.26 & 0.23 & 59.15 \\
\hline 60 & 13.84 & 2 & 1. 26 & $\odot .09$ & 60.81 \\
\hline 61 & 12.04 & 2 & 1.26 & 0.09 & 65.49 \\
\hline 62 & 13.67 & 2 & 1.26 & 0.09 & 12.82 \\
\hline 63 & 9.58 & 2 & 1.26 & 0.09 & 62.24 \\
\hline 64 & 14.22 & 2 & 1.26 & 0.09 & 78.10 \\
\hline 65 & 14.44 & 2 & 1.26 & $\odot .09$ & 56.36 \\
\hline 66 & 12.21 & 2 & 1.26 & $\odot .09$ & 53.35 \\
\hline 67 & 14.50 & 2 & 1. 26 & $\odot .09$ & 41.38 \\
\hline 68 & 15.06 & 3 & 1.31 & 0.09 & 84.25 \\
\hline 69 & 14.64 & 3 & 1.31 & 0.09 & 52.54 \\
\hline 70 & 11.41 & 3 & 1.31 & 0.09 & 45.01 \\
\hline 71 & 13.71 & 2 & 1.26 & 0.21 & 56.12 \\
\hline 72 & 15.24 & 2 & 1.26 & 0.21 & 8.45 \\
\hline 73 & 11.18 & 2 & 1.26 & 0.21 & 62.25 \\
\hline 74 & 15.34 & 2 & 1.26 & 0.21 & 57.10 \\
\hline
\end{tabular}




\begin{tabular}{|c|c|c|c|c|c|}
\hline 75 & 12.42 & 2 & 1.26 & 0.21 & 85.28 \\
\hline 76 & 15.05 & 2 & 1.26 & 0.21 & 17.40 \\
\hline 77 & 15.49 & 2 & 1.26 & 0.05 & 58.95 \\
\hline 78 & 13.23 & 2 & 1.26 & 0.05 & 33.41 \\
\hline 79 & 13.75 & 3 & 1.00 & 0.04 & 43.33 \\
\hline 80 & 13.69 & 2 & 1.31 & 0.26 & 65.77 \\
\hline 81 & 15.19 & 2 & 1.31 & 0.26 & 18.46 \\
\hline 82 & 11.97 & 2 & 1.31 & 0.26 & 49.51 \\
\hline 83 & 15.47 & 2 & 1.31 & 0.26 & 10.74 \\
\hline 84 & 13.63 & 2 & 1.31 & 0.26 & 62.84 \\
\hline 85 & 15.94 & 2 & 1.31 & 0.26 & 32.74 \\
\hline 86 & 9.96 & 3 & 1.34 & 0.19 & 80.41 \\
\hline 87 & 11.26 & 3 & 1.34 & 0.19 & 73.71 \\
\hline 88 & 10.99 & 3 & 1.34 & 0.19 & 53.24 \\
\hline 89 & 15.11 & 3 & 1.34 & 0.19 & 29.92 \\
\hline 90 & 13.10 & 3 & 1.34 & 0.19 & 54.94 \\
\hline 91 & 14.48 & 3 & 1.34 & 0.19 & 53.05 \\
\hline 92 & 15.00 & 3 & 1.34 & 0.19 & 30.77 \\
\hline 93 & 9.63 & 4 & 1.39 & 0.37 & 61.37 \\
\hline 94 & 14.43 & 3 & 1.30 & 0.29 & 72.59 \\
\hline 95 & 10.55 & 2 & 1.25 & 0.05 & 83.22 \\
\hline 96 & 15.70 & 2 & 1.25 & 0.05 & 21.62 \\
\hline 97 & 15.91 & 2 & 1.25 & 0.05 & 7.67 \\
\hline 98 & 14.37 & 2 & 1.25 & 0.05 & 21.92 \\
\hline 99 & 13.12 & 3 & 1.13 & 0.28 & 35.79 \\
\hline 100 & 10.22 & 3 & 1.13 & 0.28 & 83.89 \\
\hline 101 & 15.37 & 3 & 1.13 & 0.28 & 46.04 \\
\hline Mean & Dpar & $=1.26$ & & & \\
\hline Mean & Dper & $=0.17$ & & & \\
\hline \multicolumn{6}{|c|}{ Mean length $(u m)=13.60+/-0.18$} \\
\hline \multirow{2}{*}{\multicolumn{6}{|c|}{ Std. Dev. $\quad($ um $)=1.84$}} \\
\hline \multicolumn{4}{|c|}{ Skewness $\quad=-0.75$} & & \\
\hline Kurto & sis & $=0.08$ & & & \\
\hline
\end{tabular}




\section{APPENDIX C: FISSION-TRACK LABORATORY PROCEDURES}

\section{GENERAL}

Laboratory procedures (mineral separation and grain mounting) were performed at the Apatite to Zircon, Inc (A2Z). facilities located in Viola, Idaho. Paul B. O'Sullivan at the A2Z office located in Moscow, Idaho performed analytical procedures. All laser ablation of samples for age analyses were performed under the direction of Paul B. O'Sullivan using the facilities available at the Washington State University School of Earth and Environmental Sciences GeoAnalytical Laboratory in Pullman, Washington.

A general discussion of the methods undertaken to process and analyze samples by A2Z is presented below. Please see Donelick and others (2005), for a complete and detailed discussion of these particular methods and their justification.

\section{Sample Preparation and Measurement Feasibility Assessment}

Each sample was reduced to sand-sized particles using a jaw-crusher, sieved through a $300 \mu \mathrm{m}$ mesh, washed in water to remove clay-sized particles, and dried at room temperature. After drying, any apatite initially present in each sample was isolated using standard gravimetric and magnetic mineral separation techniques.

For each sample subjected to apatite fission-track analysis (AFT), at least one $1 \mathrm{~cm}^{2}$ apatite grain mount, consisting of some quantity of apatite grains immersed in epoxy resin, was prepared. Each grain mount was subsequently cured at $90^{\circ} \mathrm{C}$ for 1 hour. Once cured, each grain mount was polished to a glass-like finish to expose internal surfaces of the apatite grains present. After polishing, the apatite grain mounts were immersed in $5.5 \mathrm{~N} \mathrm{HNO}_{3}$ for 20.0 seconds $\left( \pm 0.5\right.$ seconds) at $21^{\circ} \mathrm{C}\left( \pm 1^{\circ} \mathrm{C}\right)$ to reveal all natural fission tracks that intersected the respective polished apatite grain surface.

The feasibility of measurement of the apatite fission-track parameters (i.e., grain ages and track lengths) was assessed by quickly scanning the polished and etched grain mount to determine if any dateable apatite grains were present. If more than one dateable grain was observed during this quick scan, measurement of the fission-track parameters was considered feasible.

\section{Generalized Fission-Track Procedures - AfT}

For AFT analysis, the polished grain mounts were first immersed in $5.5 \mathrm{~N} \mathrm{HNO}_{3}$ for 20.0 seconds $( \pm 0.5$ seconds) at $21^{\circ} \mathrm{C}\left( \pm 1^{\circ} \mathrm{C}\right)$ to reveal only the natural fission tracks intersecting the grain surfaces (Figure C.1). 

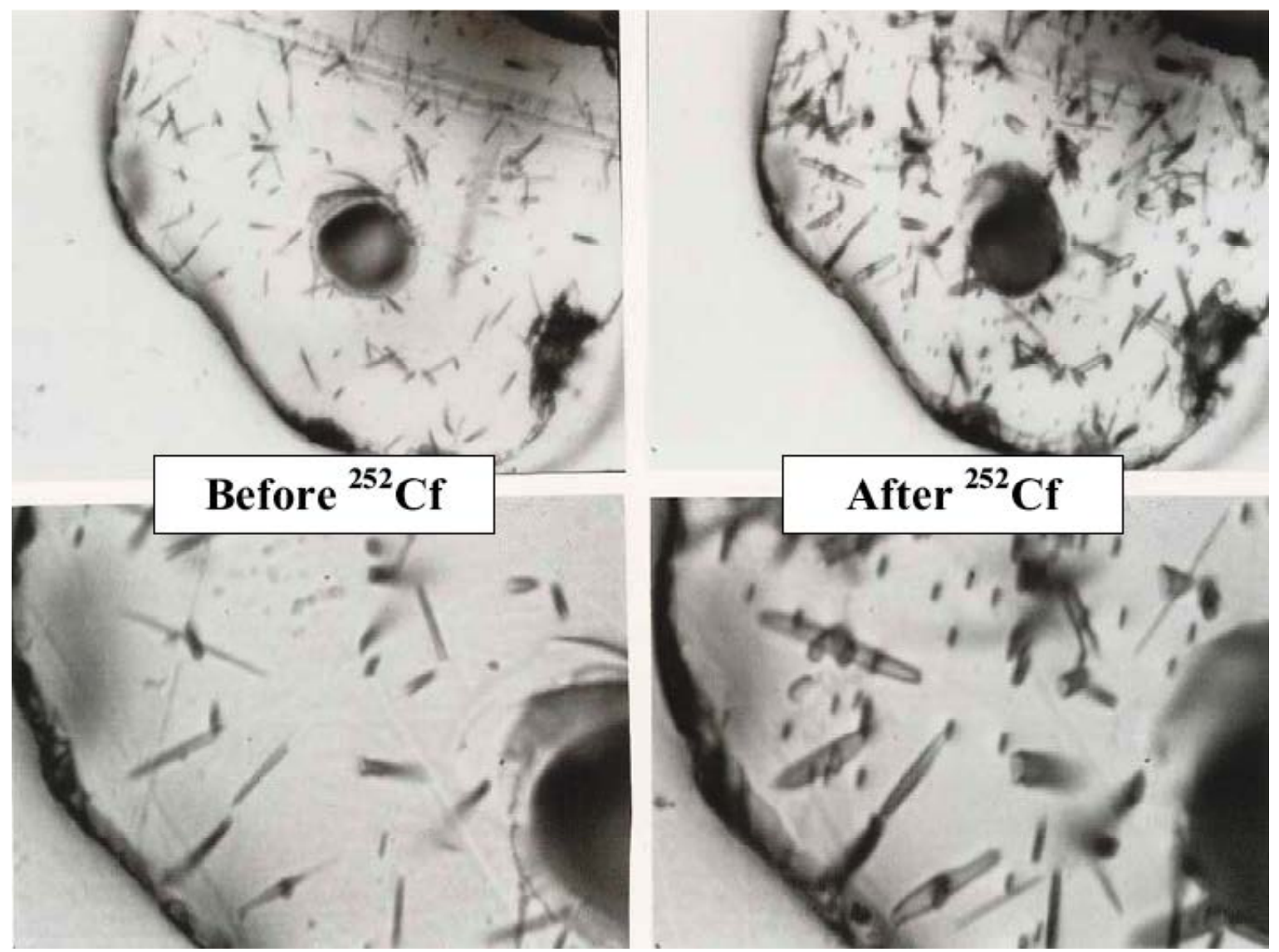

FIGURE C.1. BEFORE AND AFTER IMAGES OF APATITE GRAINS SUBJECTED TO ${ }^{252}$ CF-DERIVED FISSION-FRAGMENT IRRADIATION. UPPER IMAGES ARE AT 625X MAGNIFICATION; LOWER IMAGES ARE OF THE SAME GRAIN AT 1562X MAGNIFICATION. NOTE THE OBVIOUS INCREASE IN CONFINED TRACKS FOR LENGTH MEASUREMENT. THE LARGE PIT REPRESENTS A LASER ABLATION PIT CREATED DURING LA-ICP-MS ANALYSIS FOR AFT AGE DATING.

The mounts were then scanned to search for suitable apatite grains for age dating and those grain locations digitally recorded. For each suitable grain, representative kinetic parameters (Dpar) were measured, and the natural fission-track densities were counted. These grain localities were then revisited using the LA-ICP-MS where spot analyses to determine the concentration of $U$ were completed on the identical areas of each grain from which the natural fission-track densities were first counted. The grain mounts were then irradiated with approximately $10^{7}$ tracks $/ \mathrm{cm}^{2}$ fission fragments from a $50 \mu \mathrm{Ci}$ (activity as of July, 1996) ${ }^{252} \mathrm{Cf}$ source in a vacuum chamber. Irradiated grain mounts were then re-immersed in $5.5 \mathrm{~N} \mathrm{HNO}_{3}$ for 20.0 seconds $\left( \pm 0.5\right.$ seconds) at $21^{\circ} \mathrm{C}\left( \pm 1^{\circ} \mathrm{C}\right)$ to reveal any horizontal, confined fission tracks, and the track lengths were then measured (Figure C.1).

During analyses, all fission-track kinetic-parameter measurements, spontaneous track counts and confined tracklength measurements were completed using unpolarized light at 2000x magnification (100x dry objective, 1.25x projection tube, 16x oculars). When possible, each AFT analysis includes either 40 single-grain ages and 200 confined track length measurements.

\section{Kinetic Classification of each Apatite Grain}

It is well known that apatite fission track ages and total etched fission track lengths are strongly correlated with the solubility of their host apatite grain in samples that have experienced significant residence time at temperatures 
above approximately $70^{\circ} \mathrm{C}$ (see Burtner and others, 1994; U.S. Patent Number 5,267,274; Australian Patent Number 658,800). The parameter used to quantify solubility is termed Dpar in this report. Dpar refers to the maximum diameter of fission track etch pits parallel to the crystallographic c-axis at their intersection with the polished and etched apatite surface (Figure C.2).

Fission tracks in apatite grains exhibiting the smallest Dpar values usually anneal more quickly than fission tracks in apatite grains having larger Dpar values. In an apatite grain having a Dpar value near $1.50 \mu \mathrm{m}$ (a typical fluorinerich apatite), fission tracks generally do not survive geological heating above about $100^{\circ} \mathrm{C}$. On the other hand, in an apatite grain having a Dpar value near $3.00 \mu \mathrm{m}$ (a typical chlorine-rich apatite), fission tracks may survive geological heating above $150^{\circ} \mathrm{C}$.

For each apatite grain from which fission-track data were measured, between one and four etch pit diameters were measured and an arithmetic mean Dpar value calculated from these individual values.

(a)

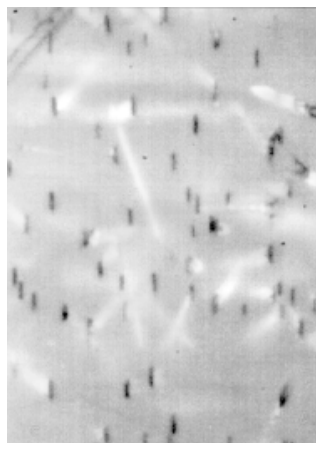

c-axis (b)

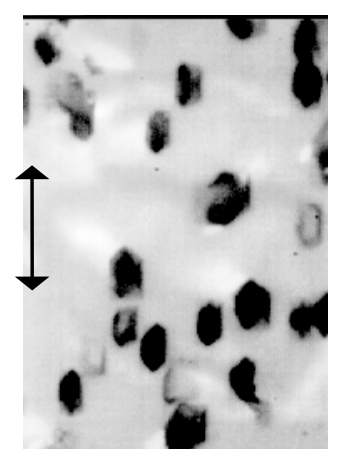

FIGURE C.2. REFLECTED LIGHT PHOTOMICROGRAPHS TAKEN AT 1562.5X MAGNIFICATION OF TWO APATITE CRYSTALS THAT EXHIBIT VERY DIFFERENT DPAR VALUES (SEE TEXT). BOTH APATITE CRYSTALS WERE ETCHED IN 5.5 $\mathrm{HNO}_{3}$ FOR 20.0 SECONDS $\left( \pm 0.5\right.$ SECONDS) AT $21^{\circ} \mathrm{C}\left( \pm 1^{\circ} \mathrm{C}\right)$. (a) DPAR=1.83 $\mu \mathrm{M}$ (APATITE FROM DURANGO, MEXICO ([F]=3.33 WT.\%, $[C L]=0.43$ WT.\%). (b) DPAR=4.99 $\mu \mathrm{M}$ (APATITE FROM BAMBLE, NORWAY ([F]=0.08 WT.\%, [CL]=6.37 WT.\%).

\section{Fission-Track Age Measurement for Apatite}

Natural fission tracks form as a result of the spontaneous nuclear fission of trace amounts of ${ }^{238} \mathrm{U}$ within an apatite grain. Using a modified version of the radioactive decay equation, the fission-track age of an apatite grain can be calculated using the ratio of the number of fission tracks intersecting the surface over a unit area in the grain to the amount of ${ }^{238} \mathrm{U}$ present in the grain.

Spontaneous tracks were first counted on suitable grain surfaces using unpolarized light at 2000x magnification. A laser ablation inductively coupled plasma-mass spectrometer (LA-ICP-MS) was then used to determine the ${ }^{238} \mathrm{U}$ concentrations by measuring the ratio of ${ }^{238} \mathrm{U}$ to ${ }^{43} \mathrm{Ca}$, from the exact regions on the individual grains from which the spontaneous tracks were initially counted (see Hasebe and others, 2004; Donelick and others, 2005). All LAICP-MS analyses were carried out at the GeoAnalytical Laboratory, Washington State University in Pullman, Washington, using the conditions and parameters outlined in Table C.2.

The fundamental assumption is made that Ca occurs in stoichiometric amounts in all apatite grains analyzed by the LA-ICP-MS. The isotope ${ }^{43} \mathrm{Ca}$ is used as the indicator of the volume of apatite ablated. Samples are ablated in a helium atmosphere to reduce condensation and elemental fractionation. 
Spot analyses, with the laser centered on a fixed point, were used (see Figure C.1). A total of 30 scans for ${ }^{238} \mathrm{U}$, ${ }^{232} \mathrm{Th},{ }^{147} \mathrm{Sm}$ and ${ }^{43} \mathrm{Ca}$ were performed for each spot analyzed. Of these scans, approximately 10 were performed while the laser was warming up and was blocked from contacting the grain surface. During this time, background counts were collected. Once the laser was permitted to hit the grain surface, a cylindrical pit was excavated to a depth beyond which uranium did not contribute fission tracks to the etched grain surface. Between 15 and 20 scans performed during pit excavation were required to reach this depth. The depths of a representative number of these pits were measured and the ${ }^{238} \mathrm{U} /{ }^{42} \mathrm{Ca}$ value for the pit as a whole was determined based on the weighted mean of the ${ }^{238} \mathrm{U} /{ }^{43} \mathrm{Ca}$ value for individual scans relative to the depths from which the ablated material was derived.

TABLE C.2. ICP-MS AND LASER OPERATING CONDITIONS AND DATA ACQUISITION PARAMETERS.

\begin{tabular}{|c|c|}
\hline \multicolumn{2}{|c|}{ ICP-MS: operating conditions } \\
\hline Instrument & Finnigan Element II Magnetic Sector ICP-MS \\
\hline Forward power & $1.25 \mathrm{~kW}$ \\
\hline Reflected power & $<5 \mathrm{~W}$ \\
\hline Plasma gas & $\operatorname{Ar}$ \\
\hline Coolant flow & $15 \mathrm{l} / \mathrm{min}$ \\
\hline Carrier flow & $1.0 \mathrm{l} / \mathrm{min}(\mathrm{Ar}) 0.8 \mathrm{l} / \mathrm{min}(\mathrm{He})$ - optimized daily \\
\hline Auxiliary flow & $0.9 \mathrm{I} / \mathrm{min}$ \\
\hline \multicolumn{2}{|c|}{ ICP-MS: acquisition parameters } \\
\hline Dwell time & 18 milliseconds per peak point \\
\hline Points per peak & 4 \\
\hline Mass window & $5 \%$ \\
\hline Scans & 30 \\
\hline Data acquisition time & $22 \mathrm{sec}$ \\
\hline $\begin{array}{l}\text { Data acquisition mode } \\
\text { Isotopes measured }\end{array}$ & $\begin{array}{l}\text { electronic scanning } \\
{ }^{43} \mathrm{Ca} \text { (apatite) or }{ }^{29} \mathrm{Si} \text { (zircon), }{ }^{238} \mathrm{U},{ }^{232} \mathrm{Th} \text {, and }{ }^{147} \mathrm{Sm}\end{array}$ \\
\hline \multicolumn{2}{|c|}{ Laser: operating conditions } \\
\hline Laser type & New Wave UP213 (Nd: YAG) \\
\hline Wavelength & $213 \mathrm{~nm}$ \\
\hline Laser mode & Q switched \\
\hline Laser output power & $8 \mathrm{~J} / \mathrm{cm}$ \\
\hline Laser warm up time & $6 \mathrm{sec}$ \\
\hline Shot repetition rate & $5 \mathrm{~Hz}$ \\
\hline Sampling scheme & spot (16 $\mu \mathrm{m}$ apatite, $12 \mu \mathrm{m}$ zircon) \\
\hline
\end{tabular}

The fission track ages and errors were calculated using: (a) the ratio of the density of natural fission tracks present in the grain to the amount of ${ }^{238} \mathrm{U}$ present, and (b) a modified version of the radioactive decay equation that includes a LA-ICPMS zeta calibration factor (see equations $1 \mathrm{~b}$ for age equation and $2 \mathrm{~b}$ for error calculation in Donelick and others, 2005). This zeta calibration factor is determined for each sample analyzed during each LA-ICPMS session by analyzing the U:Ca ratio of an apatite calibration standard with known age at the beginning and end

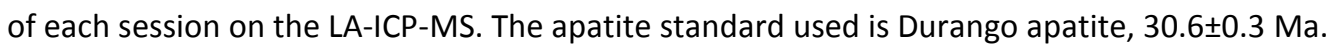

\section{Fission-Track Length Measurement for Apatite}

The total etched length of a natural fission track in apatite is a strong indicator of the integrated thermal history that the track has experienced. Fission tracks form continuously through time at a rate determined solely by the concentration of ${ }^{238} \mathrm{U}$ in the host apatite grain. As such, the distribution of fission-track lengths in an apatite 
contains abundant information about the time-temperature path experienced by the apatite, particularly the cooling history since the time of peak temperature.

Following age analysis, apatite mounts were then irradiated using fission fragments from a ${ }^{252} \mathrm{Cf}$ source in a vacuum chamber in order to enhance the number of confined tracks available for measurement (e.g. Donelick and Miller, 1991; Donelick and others, 2005). Donelick and Miller (1991) demonstrated that irradiating apatite grains with ${ }^{252} \mathrm{Cf}$-derived fission fragments could yield a 20 -fold increase in the number of available fission tracks for length measurement (Figure C.3).

(a)

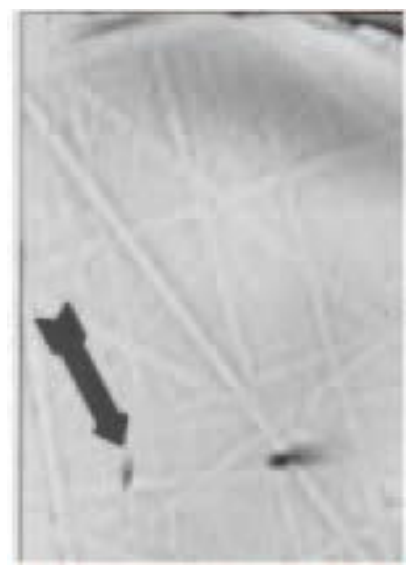

(b)

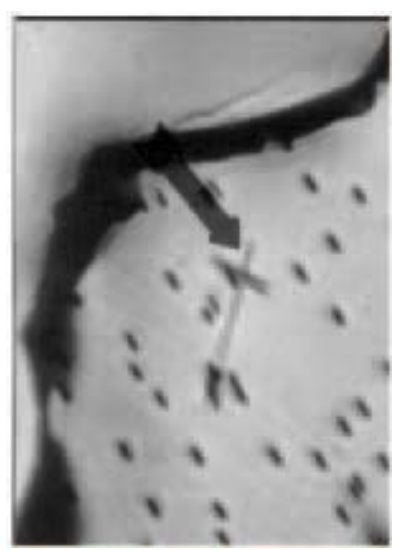

FIGURE C.3. TRANSMITTED LIGHT PHOTOMICROGRAPHS TAKEN AT 1562.5X MAGNIFICATION OF TWO APATITE CRYSTALS FROM THE SAME SAMPLE THAT EXHIBIT LOW NATURAL FISSION-TRACK DENSITIES. (a) NOT IRRADIATED WITH ${ }^{252}$ CF FISSION FRAGMENTS (ARROW INDICATES A NATURAL FISSION TRACK).

The ${ }^{252} \mathrm{Cf}$-irradiated grain mounts were then re-etched using the same formula as before in order to reveal horizontal, confined fission tracks within the apatite grains. Only natural, horizontal, confined fission tracks in apatite with clearly visible ends were considered candidates for length measurement. The length and crystallographic orientation of each fission track was determined using a digitizing tablet interfaced with a personal computer. The precision of each track length is estimated to be $\pm 0.20 \mu \mathrm{m}$; the precision of each track angle to the crystallographic c-axis is estimated to be \pm 2 degrees. 